\title{
Circuit
}

Musiques contemporaines

\section{Introduction : Cinquante ans et une Semaine}

\section{Claudine Caron et Jonathan Goldman}

Volume 21, numéro 3, 2011

Musique automatiste ? Pierre Mercure et le Refus global

URI : https://id.erudit.org/iderudit/1006356ar

DOI : https://doi.org/10.7202/1006356ar

Aller au sommaire du numéro

Éditeur(s)

Les Presses de l’Université de Montréal

ISSN

1183-1693 (imprimé)

1488-9692 (numérique)

Découvrir la revue

Citer ce document

Caron, C. \& Goldman, J. (2011). Introduction : Cinquante ans et une Semaine. Circuit, 21(3), 5-8. https://doi.org/10.7202/1006356ar d'utilisation que vous pouvez consulter en ligne.

https://apropos.erudit.org/fr/usagers/politique-dutilisation/ 


\title{
Introduction :
}

\section{Cinquante ans et une Semaine}

\author{
Claudine Caron et Jonathan Goldman
}

On a tout à gagner à avoir de l'audace...

- Pierre Mercure

Dans le volume 17, $\mathrm{n}^{\circ}$ 2, sur l'avant-garde musicale en Amérique latine, Jonathan Goldman proposait un rapprochement entre le compositeur mexicain Manuel Enríquez - avec qui Ricardo dal Farra avait réalisé un entretien publié dans le même numéro - et le compositeur québécois Pierre Mercure (1927-1966), notant qu'Enríquez, qui «était un compositeur instrumental dans l'âme, mais qui s'est livré néanmoins admirablement à l'aventure électroacoustique ${ }^{1}$ ", faisait penser à Mercure, de qui il était l'aîné d'un an seulement ${ }^{2}$. Ainsi a-t-il fallu attendre quatre ans pour que Circuit s'attarde à l'œuvre de Pierre Mercure, figure de proue de l'avant-garde musicale dans le Québec de l'après-guerre. À première vue, le catalogue d'œuvres de Pierre Mercure, de genres hétéroclites, pose en effet certaines difficultés à quiconque désire cantonner le compositeur dans une contemporanéité univoque : son parcours va de la musique tonale pour orchestre à des œuvres électroacoustiques; sa production inclut de la musique pour le cinéma, la danse et le théâtre 3 ; et la variété des supports sur lesquels se trouve sa musique entraine des défis considérables quant à la conservation et à l'accessibilité des sources.

Mercure ayant vécu au tournant d'une époque où la musique était semblet-il la parente pauvre de la création artistique au Québec, il reste à élucider les questions fondamentales sur la pensée et l'œuvre de ce compositeur au visage protéiforme, à la fois le compositeur de Kaléidoscope (1947-1948), l'une des œuvres orchestrales canadiennes les plus jouées, le réalisateur d'une émission télévisuelle comme il ne s'en produit plus, «L'Heure du concert »
1. Jonathan Goldman, "De l'autre Amérique à la nôtre : un regard déboussolant", Circuit, vol. 17, nº 2, p. 9.

2. Pas moins de vingt-huit œuvres de Pierre Mercure peuvent être écoutées sur le site du Centre de musique canadienne : http:// www.musiccentre.ca/apps/index. cfm?fuseaction=composer.FA_dsp_ sample\&authpeopleid $=1111 \& b y=M$

3. Le fonds Pierre Mercure est conservé au Centre d'archives de Montréal et comprend des partitions manuscrites, de la correspondance, des programmes, des photographies, des coupures de presse, des carnets de voyage et nombre d'autres documents inédits. À ce jour, seulement deux publications ont été consacrées au compositeur: le fascicule Compositeurs au Québec: Pierre Mercure, Louise Laplante (dir.), Centre de musique canadienne au Québec, 1976 et le livret du coffret de disques "Anthologie de la musique canadienne : Pierre Mercure", AMC 35 , $\mathrm{RCl}, 1990$. 
4. Le $50^{\mathrm{e}}$ anniversaire de la SIMA a été célébré par la Société de musique contemporaine du Québec, le Centre de musique canadienne au Québec et Le Vivier lors d'une journée d'étude intitulée "Pierre Mercure (19271966) : du Refus global à l'art total", coorganisée par Claudine Caron, Mireille Gagné et Mario Gauthier, dans le cadre du festival Montréal/Nouvelles Musiques, le 23 février 2011.

5. Paul-Émile Borduas ([1948] 1997), Refus global et autres écrits, Montréal, TYPO.

6. Les signataires de Refus global étaient : Madeleine Arbour, Marcel Barbeau, Paul-Émile Borduas, Bruno Cormier, Claude Gauvreau, Pierre Gauvreau, Muriel Guilbault, Marcelle Ferron, Fernand Leduc, Thérèse Renaud-Leduc, Jean-Paul Mousseau, Maurice Perron, Louise Renaud, Françoise Riopelle, Jean-Paul Riopelle et Françoise Sullivan.

7. Affiche de la conférence présentée par Lyse Richer au studio du Musée d'art contemporain (Montréal), le 14 mars 1976, fonds Pierre Mercure.
(1954-1966), ou encore l'instigateur d'un événement qui allait marquer l'histoire de la musique au pays, la Semaine internationale de musique actuelle (SIMA), dont nous soulignons ici le $50^{\mathrm{e}}$ anniversaire ${ }^{4}$. Cet événement d'envergure, présenté dans le cadre des Festivals de Montréal du 3 au 8 août 1961, permettait effectivement au public d'entendre des œuvres de compositeurs tels que Brown, Cage, Feldman, Kagel, Nono, Schaeffer, Stockhausen ou Varèse, et se démarquait par le nombre de compositeurs et d'artistes nordaméricains qui figuraient au programme, ainsi que par les étonnantes collaborations qu'il proposait entre des musiciens, des artistes plastiques et des chorégraphes de premier plan qui y ont donné des performances.

Lors d'une conférence présentée en 1976, Lyse Richer confrontait l'œuvre de Mercure aux idéaux de Refus global (1948)5 , titre du manifeste artistique qui allait mettre en avant la liberté de création, l'abstraction et la spontanéité, donnant naissance à l'automatisme, ce mouvement que l'on associe à PaulÉmile Borduas et aux quinze autres signataires ${ }^{6}$ et qui a notamment donné l'impulsion aux célèbres toiles dont l'une des plus notoires orne la couverture de ce numéro. Même si à la question de Richer «Pierre Mercure fait-il de l'automatisme en musique? ?», dont une allusion reste à même le titre de ce numéro, il faut répondre par la négative, la poser à nouveau rouvre la porte à la compréhension de l'interdisciplinarité qui prévaut dans l'œuvre de Mercure. Il serait sans doute encore hasardeux de tenter d'assimiler Mercure aux automatistes. D'une part, le musicien ne figure pas parmi les signataires de Refus global; d'autre part, les œuvres pour orchestre de Mercure peuvent difficilement être assimilées à l'esprit de spontanéité au cœur de la démarche automatiste. Toutefois, il n'est pas anodin que Mercure ait côtoyé et créé aux côtés des Gauvreau, Mousseau, Riopelle et Sullivan parmi tant d'autres, et il va sans dire qu'il y a eu entre eux de l'émulation, puis une ouverture de l'univers des possibles. En effet, des recherches récentes effectuées par des spécialistes de différents domaines - de la musicologie à l'histoire de l'art et de la danse - permettent de saisir l'affinité éloquente qui relie l'esprit de création déclenché par le Refus global - ce monument de la littérature québécoise - à la démarche de Mercure, ainsi qu'à la musique en général.

Issus de points de vue divers, les articles dans ce numéro apportent autant d'éléments de réponse sur la place de la musique dans le cercle des automatistes, sur les aspects automatistes de l'œuvre de Pierre Mercure et sur les liens qu'il a entretenus avec les protagonistes de Refus global. Alors que la musicologue Claudine Caron, codirectrice de ce numéro, parcourt la production musicale considérable de Mercure diffusée dans les spectacles de danse (œuvres chorégraphiées par des sommités de la danse moderne 
naissante au Québec telles que Françoise Sullivan, Jeanne Renaud et Françoise Riopelle), Allana C. Lindgren, historienne de la danse, repense les rapports entre la danse et la musique dans l'œuvre chorégraphique de Jeanne Renaud et de Françoise Sullivan. Pour sa part, l'historien de l'art Gilles Lapointe raconte la pré-histoire de cet opéra inabouti qui aurait relié le poète Claude Gauvreau à Pierre Mercure autour du thème pour le moins séduisant du Vampire et la nymphomane, un texte qui, des décennies plus tard, a été mis en musique par deux autres compositeurs : Serge Provost (1992) et Claude Lassonde (1996) ${ }^{8}$. En tant que compositeur, Pierre Mercure a été profondément marqué par la musique expérimentale de John Cage, une attirance qui s'observe dans une œuvre aléatoire comme $\mathrm{H}_{2} \mathrm{O}$ per Severino (1965), ainsi que dans la programmation de la SIMA, qui donnait la création mondiale de la monumentale Atlas eclipticalis du compositeur américain. Sophie Stévance voit une parenté dans ce parti pris esthétique à travers le genre de la musique actuelle, que nos lecteurs connaissent bien depuis la parution du vol. 6, n ${ }^{\circ}$ 2. Dans son enquête, Stévance suit la trajectoire sémantique du terme «musique actuelle » qui l'amènerait de la SIMA jusqu'au Festival international de musique actuelle de Victoriaville (FIMAV), haut lieu de la diffusion de la musique de Jean Derome, Joane Hétu, Michel F. Côté, Productions SuperMusique et des autres défenseurs de ce courant. Mais Pierre Mercure ne s'est pas seulement nourri auprès des pratiquants américains de la musique expérimentale. Les avant-gardes musicales européennes ont retenu son attention longuement et il a notamment fait le pèlerinage - comme tant d'autres - aux cours internationaux de Darmstadt. Dans le prolongement du vol. $15, \mathrm{n}^{\circ} 3$ ( Souvenirs de Darmstadt»), le musicologue Jean Boivin documente la présence de plusieurs Canadiens à Darmstadt - dont Mercure -, et ce, déjà depuis le début des années 1950. À la lecture de son article, nous entrevoyons un champ canadien de la musique contemporaine construit de divers éléments géographiquement espacés. Dans la rubrique Document, l'artiste sonore et chercheur indépendant Mario Gauthier présente la transcription d'une table ronde radiophonique, réalisée par Maryvonne Kendergi en pleine SIMA (1961), qui réunissait Pierre Mercure et plusieurs autres acteurs importants de la vie musicale montréalaise de l'époque. Enfin, vous ne serez pas étonnés d'apprendre que le Cahier d'analyse porte sur des œuvres de Pierre Mercure; le compositeur Brian Cherney ausculte les œuvres orchestrales Triptyque et Lignes et points.

Si les auteurs de ce numéro ne prétendent pas répondre définitivement à la question de l'affinité automatiste au sein de la démarche de Pierre Mercure, il est hors de tout doute raisonnable de penser que ce compositeur
8. À propos de l'opéra de Provost, voir le vol. 3, nº 2 (1992) de Circuit, "Opéra? Gauvreau, Provost, Kagel". 
entreprit chacun de ses projets avec une liberté en phase avec les tendances des artistes qu'il a côtoyés, signataires ou non de Refus global, de Jeanne Renaud à Armand Vaillancourt, de Pierre Moretti à John Cage. En terminant les corrections de ce numéro, nous apprenions le décès de Madame Maryvonne Kendergi (1915-2011), qui nous a quittés le 27 septembres dernier. Ce numéro est dédié à sa mémoire.

\section{Bonne lecture!}

\section{Montréal, octobre 2011}

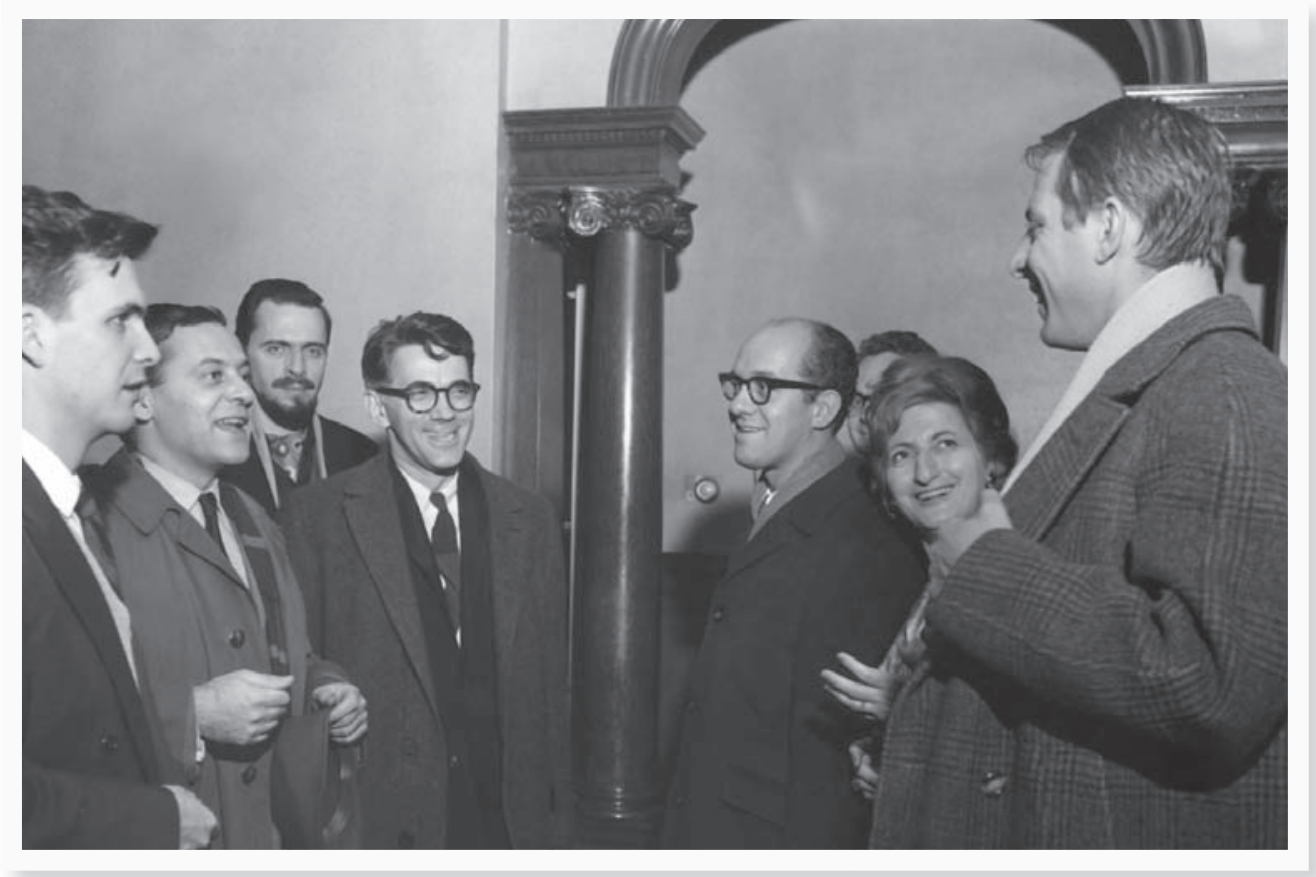

Musiciens réunis chez Gilles Tremblay après un concert de la musique de Stockhausen donné à la salle Saint-Sulpice, à Montréal, en janvier 1964. De gauche à droite : Gilles Tremblay, Pierre Mercure, Guy Lachapelle, David Tudor, Serge Garant, István Anhalt (partiel), Maryvonne Kendergi et Karlheinz Stockhausen. 